\title{
SCIENTIFIC REPORTS

\section{OPEN A novel hotspot specific isothermal amplification method for detection of the common PIK3CA p.H1047R breast cancer mutation}

\author{
Melpomeni Kalofonou $\left(\mathbb{1}^{1,4^{*}}\right.$, Kenny Malpartida-Cardenas ${ }^{1,4}{ }^{1,4}$, George Alexandrou ${ }^{1,4}$, \\ Jesus Rodriguez-Manzano ${ }^{1}$, Ling-Shan $\mathrm{Yu}^{1}$, Nicholas Miscourides ${ }^{1}$, Rebecca Allsopp ${ }^{2}$, \\ Kelly L. T. Gleason ${ }^{3}$, Katie Goddard ${ }^{3}$, Daniel Fernandez-Garcia ${ }^{2}$, Karen Page ${ }^{2}$, \\ Pantelis Georgiou $\mathbb{D}^{1}$, Simak Ali $\mathbb{D}^{3}$, R. Charles Coombes ${ }^{3}$, Jacqueline Shaw $\mathbb{D}^{2}$ \& \\ Christofer Toumazou ${ }^{1}$
}

Breast cancer (BC) is a common cancer in women worldwide. Despite advances in treatment, up to $30 \%$ of women eventually relapse and die of metastatic breast cancer. Liquid biopsy analysis of circulating cell-free DNA fragments in the patients' blood can monitor clonality and evolving mutations as a surrogate for tumour biopsy. Next generation sequencing platforms and digital droplet PCR can be used to profile circulating tumour DNA from liquid biopsies; however, they are expensive and time consuming for clinical use. Here, we report a novel strategy with proof-of-concept data that supports the usage of loop-mediated isothermal amplification (LAMP) to detect PIK3CA c.3140 A $>$ G (H1047R), a prevalent $B C$ missense mutation that is attributed to $B C$ tumour growth. Allele-specific primers were designed and optimized to detect the p.H1047R variant following the USS-sbLAMP method. The assay was developed with synthetic DNA templates and validated with DNA from two breast cancer cell-lines and two patient tumour tissue samples through a QPCR instrument and finally piloted on an ISFET enabled microchip. This work sets a foundation for $\mathrm{BC}$ mutational profiling on a Lab-on-Chip device, to help the early detection of patient relapse and to monitor efficacy of systemic therapies for personalised cancer patient management.

Breast cancer (BC) has a lifetime incidence risk of $12 \%$, with an overall survival of more than $70 \%$ of reported cases when early detection is possible ${ }^{1,2}$. Although surgery is capable of removing the primary tumour, minimal residual disease (MRD)/micrometastases may persist resulting in eventual resistance to therapy and recurrence ${ }^{3}$. MRD can often persist even after adjuvant therapy and can grow and spread overtime, remaining undetectable through mammograms, MRI scans and current tumour marker blood tests, such as CA-15-3 and CA 27.29 antigen assays ${ }^{4}$. These current tumour marker blood tests work as a cost-effective method to measure disease progression but do not provide information about mutational changes and heterogeneity of the primary tumour or MRD. With the development of new, non-cross-resistant treatments for breast cancer, early detection of $\mathrm{MRD} / \mathrm{micro-}$ metastases and mutational profiling of circulating tumour DNA (ctDNA) provides an attractive, cost-effective alternative approach to the detection of early signs of relapse and treatment switching.

Previous research has proven that circulating free DNA (cfDNA) contains DNA fragments from cancer cells (ctDNA) that are released in blood, showing somatic mutations that reflect the original cancer and evolved clonal subtypes $^{5}$. In particular, recent efforts have established that hotspot mutations in PIK3CA, a gene mutated in $20-40 \%$ of metastatic BC, are also commonly observed in screen-detected stage- $1 \mathrm{BCs}^{6}$. The PI3K protein is involved in the AKT/mTOR pathway and, when deregulated, leads to tumour-cell growth ${ }^{7}$. Investigation has

${ }^{1}$ Centre for Bio-Inspired Technology, Department of Electrical and Electronic Engineering, Imperial College London, London, SW7 2AZ, England. 'Leicester Cancer Research Centre, Department of Genetics and Genome Biology, University of Leicester, Leicester, LE2 7LX, England. ${ }^{3}$ Division of Cancer, Department of Surgery and Cancer, Imperial College London, London, SW7 2AZ, England. ${ }^{4}$ These authors contributed equally: Melpomeni Kalofonou, Kenny Malpartida-Cardenas and George Alexandrou. *email: m.kalofonou@imperial.ac.uk 


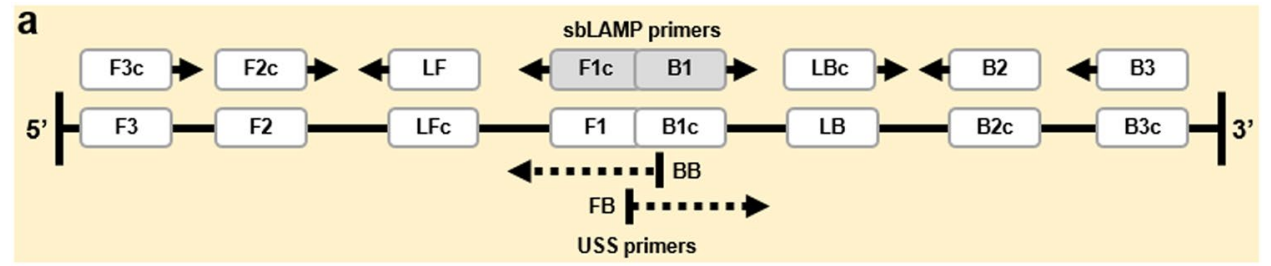

b
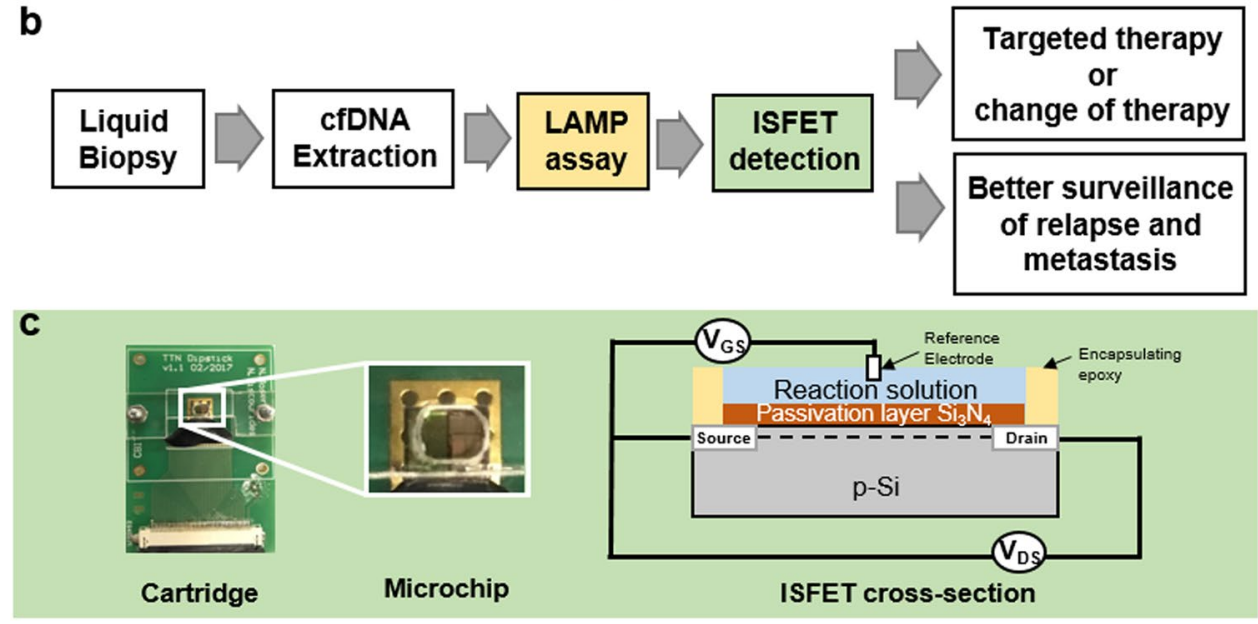

Figure 1. Schematic of the SNV-based LAMP method, proposed workflow and cartridge of CMOS microchip. (a) Representation of the SNV-based LAMP method including USS primers that prevent unspecific amplification $^{15}$. (b) Proposed workflow showing how the protocol described here can be implemented with liquid biopsies extraction to patient's treatments and relapses management. (c) Lab-on-Chip platform showing the PCB cartridge, microchip, microfluidic chamber and cross-section of an ISFET sensor ${ }^{16}$.

indicated that the PIK3CA p.H1047R missense mutation causes a constitutively active form of the PI3K protein and is associated with poor prognosis and BC disease progression ${ }^{8}$. Detecting BC mutations from ctDNA has been successful with next-generation sequencing workflows like BEAMing ${ }^{9-11}$ or digital droplet PCR (ddPCR) but cannot be easily translated into the clinic due to multifactorial reasons such as requirement of clear prognostic value, expensive costs and specialised labour per sample $e^{5,12,13}$.

To circumvent this requirement of expensive thermal-cycling machinery of PCR technology and sequencing workflows, isothermal amplification was investigated as an alternative method of detection. Loop-mediated isothermal amplification (LAMP) developed by Notomi et al. ${ }^{14}$ uses 6 template specific primers to amplify DNA with high specificity and sensitivity at a constant temperature. LAMP has often been used in the study of pathogens and infectious diseases but has recently started to be used for mutational detection. A variation of the LAMP protocol using USS-sbLAMP primers ${ }^{15}$ can help discriminate single base mutational detection allowing cancer variants to be investigated by LAMP, as shown in Fig. 1a. These properties of LAMP coupled with its higher amplification yield allows it to be utilised in tandem with standard microchip technology used for chemical sensing such as the Ion-Sensitive Field-Effect Transistors (ISFETs) ${ }^{16}$. ISFETs fabricated in unmodified CMOS technology have been already described to detect DNA targets via the $\mathrm{pH}$ change induced during positive amplification results ${ }^{17-23}$. ISFET-based DNA detection in combination with LAMP's ability to run isothermally allows the technology to be applied at the point-of-care as a Lab-on-Chip (LoC) device.

In this study, novel primer sets for the detection of PIK3CA p.H1047R variant in isothermal conditions have been developed, enabling fast, sensitive and specific detection of wild type (WT) and mutant (MT) p.H1047R alleles, while differentiating the detectable signals in an environment of interfering DNA templates. Time-to-results below 25 minutes with a limit of detection of $10^{3}$ copies per reaction show the potential of adapting this assay to the clinic for rapid and sensitive profiling of common BC mutations. Besides lab-based validation, we demonstrate on-chip specific detection of both WT and MT alleles using an ISFET-based Lab-on-Chip platform. Using this fully-electronic chemical sensing technology in combination with the molecular methods developed here, we are in a position to create a lab-free, cost-effective sample-to-result system for routine monitoring of a panel of breast cancer mutations through liquid biopsy profiling of ctDNA, to be used as a diagnostic companion to stratify patient treatment. Future adoption would lead to significant advantages, such as offering continuous monitoring of tumour progression while assisting imaging intervention and tumour localisation, aiming to adaptively individualise breast cancer treatment for patient benefit. The technology would also be primed to monitor MRD post-surgery and help proactively search for therapy resistance mutations during a patients' treatment regime. The proposed workflow of the system and the Lab-on-Chip platform used to demonstrate feasibility of the SNV-based LAMP method are demonstrated in Fig. 1(b,c). 


\begin{tabular}{|c|c|}
\hline Primer ID & Sequence $\left(5^{\prime} \rightarrow 3^{\prime}\right)$ \\
\hline H1047R_F3 & AGA ACT ACA ATC TTT TGA TGA CA \\
\hline H1047R_B3 & TGG AAT CCA GAG TGA GCT \\
\hline H1047R_LF & AAT ACT CCA AAG CCT CTT GCT C \\
\hline H1047R_LB & TGG ATC TTC CAC ACA ATT AAA CAG C \\
\hline H1047R_sbFIP $19 \_W T$ & TGT GCA TCA TTC ATT TGT TGC ATA CAT TCG AAA GAC CCT \\
\hline H1047R_sbBIP $14 \_W T$ & ATC ATG GTG GCT GGC TCA GTT ATC TTT TCA GTT CAA TG \\
\hline H1047R_sbFIP $17 \_$MT & CGT GCA TCA TTC ATT TGG CAT ACA TTC GAA AGA CCC T \\
\hline H1047R_sbBIP $14 \_M T$ & GTC ATG GTG GCT GGC TCA GTT ATC TTT TCA GTT CAA TG \\
\hline H1047R_FB2 $21 \_$MT & ACG TCA TGG TGG CTG GAC AAC AA \\
\hline H1047R_BB2 24_MT & GAC GTG CAT CAT TCA TTT GTT TCA TG \\
\hline H1047R_FB2 ${ }_{19 \_}$WT & ACA TCA TGG TGG CTG GAC AAC \\
\hline H1047R_BB1 $1_{23}$ WT & ATG TGC ATC ATT CAT TTG TTT CAT \\
\hline
\end{tabular}

Table 1. USS-sbLAMP primer sequences for allele-specific amplification of SNV p.H1047R.

\section{Results}

SNV-specific LAMP for detection of p.H1047R. Specific amplification of the PIK3CA p.H1047R gene mutation in isothermal conditions was achieved following the USS-sbLAMP method described by MalpartidaCardenas et al. ${ }^{15}$. This method consisted of (i) Single Nucleotide Variant (SNV) LAMP (sbLAMP) primers in charge of allele-specific amplification, and (ii) unmodified self-stabilising (USS) competitive primers responsible for preventing unspecific amplification. The sbLAMP primers included the LAMP priming regions F3, B3, LF, LB and the allele-specific primers sbFIP and sbBIP. These last two primers contained the regions F1c-F2 and B1c$\mathrm{B} 2$, respectively. Considering the GC\% content of the target sequence, different lengths of F1c and B1c priming regions were designed for optimal performance at $63^{\circ} \mathrm{C}$ and screened experimentally; due to the higher GC $\%$ content at the region where $\mathrm{B} 1 \mathrm{c}$ is located, the final design consisted of B1c being shorter than F1c to enhance allele-specificity without hampering the analytical sensitivity.

Specifically, the sbLAMP primer set targeting the mutant (MT) allele, named as sbLAMP ${ }_{\mathrm{MT}}$, included sbFIP 17- $_{-}$ MT and sbBIP ${ }_{14}$ MT primers. On the other hand, the sbLAMP primer set targeting the wild type (WT) allele, named as sbLAMP ${ }_{\mathrm{WT}}$, consisted of sbFIP ${ }_{19}$ WT and sbBIP ${ }_{14-}$ WT primers. USS primers were designed manually based on the aforementioned sbFIP and sbBIP primers. USS primers were incorporated in the reaction as follows: MT reaction included sbLAMP ${ }_{\mathrm{MT}}$ with $\mathrm{USS}_{\mathrm{WT}}$, and WT reaction included sbLAMP ${ }_{\mathrm{MT}}$ with USS $\mathrm{WT}_{\mathrm{WT}}$. Different lengths and concentrations were tested following the guidelines described ${ }^{15}$. The final primer sets consisted of $\mathrm{USS}_{\mathrm{MT}} \mathrm{FB} 2_{21} \mathrm{MT} / \mathrm{BB} 2_{24} \mathrm{MT}$ and $\mathrm{USS}_{\mathrm{WT}} \mathrm{FB} 2_{19} \_\mathrm{WT} / \mathrm{BB} 1_{23} \_\mathrm{WT}$, both at a concentration of $3 \mu \mathrm{M}$ per reaction. Primer sequences are shown in Table 1.

Analytical sensitivity and specificity of SNV-specific LAMP. Analytical sensitivity of the designed LAMP primer sets was evaluated with ten-fold serial dilutions of synthetic plasmid DNA at $1 \times 10^{7}, 1 \times 10^{6}, 1 \times$ $10^{5}, 1 \times 10^{4}$ and $1 \times 10^{3}$ copies/reaction. Two reactions, WT and MT reactions, were performed independently. WT and MT templates were tested in both reactions to assess the analytical sensitivity and specificity of the designed USS-sbLAMP primer sets. Both templates were uniquely amplified within their corresponding specific reactions at concentrations below $1 \times 10^{6}$ copies/reaction with a limit of detection of $1 \times 10^{3}$ copies/reaction within $25 \mathrm{~min}$. In Fig. 2a, standard curves are presented showing coefficient of determinations $\left(\mathrm{R}^{2}\right)$ of 0.992 and 0.972 for the specific amplification of the WT and MT template, respectively. These results showed the capability of the assay for sample quantification. Both WT and MT reactions presented a linear working range from $1 \times$ $10^{7}$ to $1 \times 10^{3}$ copies/reaction. A complex and more realistic genomic environment was created by incorporating Salmon testes sonicated DNA in the reaction mixture as background DNA. Results presented in Fig. 2b show standard curves of specific WT and MT reactions. Remarkably, the limit of detection $\left(1 \times 10^{3}\right.$ copies/reaction) and the linear working range $\left(1 \times 10^{7}\right.$ to $1 \times 10^{3}$ copies/reaction $)$ were preserved in this scenario without any significant variation in the TTP values (correlation of 0.98 and 0.99 for each specific reaction, according to statistical linear regression), as shown in Table 2.

Accuracy of detection in a mixture of allele ratios. Allele discrimination within allelic ratios (100/0, $80 / 20,50 / 50,20 / 80$, and 0/100, in percentages) in spiked mixed populations was studied. This scenario included Salmon testes sonicated background DNA with the results shown in Fig. 3. Both alleles were discriminated by combining the results of the WT and MT reactions, denoting the high specificity of the assay to distinguish each allele within mixed populations. Reactions targeting the allele that was not present in the mixed population, such as the ratios 100/0 and 0/100, did not show any amplification. The TTP values obtained clearly fitted in the corresponding standard curves previously built for each allele (Fig. 2b), and therefore, the capability of DNA quantification within a mixed population was demonstrated. Calculated concentrations in copies/reaction and the calculated ratios are presented in Fig. 3b. Overall, these results show the feasibility of the developed assay to estimate copy number and ratios with high accuracy.

Validation with positive and negative DNA samples derived from patient tissue and cell line controls. DNA from MCF7, T47D cell lines (Fig. 4a-c) and from Formalin-Fixed Paraffin-Embedded (FFPE) 

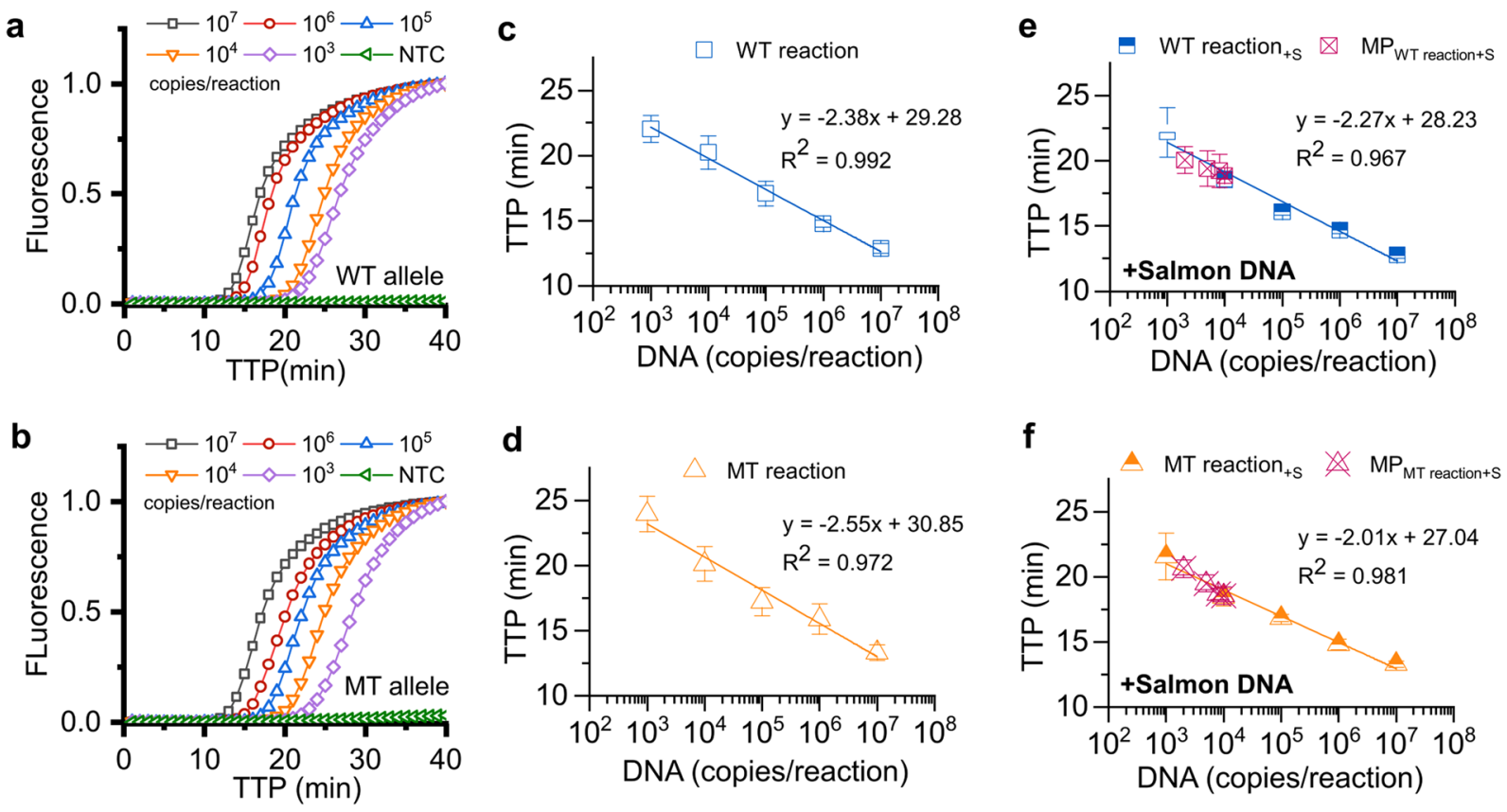

Figure 2. Amplification and standard curves of USS-sbLAMP reactions for detection of SNV p.H1047R utilising a synthetic plasmid DNA template containing a $531 \mathrm{bp}$ fragment of the gene region. (a) Amplification curves of the WT template at different DNA concentrations ranging from $10^{7}$ to $10^{3}$ copies/reaction $(31.356 \mathrm{pg} /$ reaction to $0.003 \mathrm{pg} /$ reaction respectively) using the WT reaction. (b) Amplification curves of the MT template at different DNA concentrations ranging from $10^{7}$ to $10^{3}$ copies/reaction $(31.356 \mathrm{pg} /$ reaction to $0.003 \mathrm{pg} /$ reaction respectively) using the MT reaction. (c) Standard curve of the DNA template harbouring the WT allele, showing linear fitting equation and coefficient of determination. (d) Standard curve of the DNA template harbouring the MT allele, showing linear fitting equation and coefficient of determination. (e) Standard curve of the DNA template harbouring the WT allele with the addition of background Salmon testes sonicated DNA at $13 \mathrm{ng} /$ reaction $(+S)$. Results from the specific amplification of mixed populations are depicted in dark pink and labelled as $\mathrm{MP}_{\mathrm{WT} \text { reaction }+\mathrm{s}}$. (f) Standard curve of the DNA template harbouring the MT allele with the addition of background Salmon testes sonicated DNA at $13 \mathrm{ng} /$ reaction $(+\mathrm{S})$. Results from the specific amplification

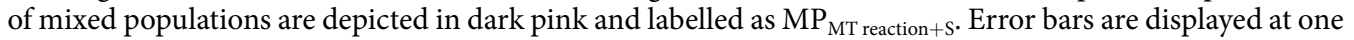
standard deviation. Reactions were considered as negative at time-to-positive (TTP) above 25 min. No template control (NTC) was included. Average of two experiments performed in triplicates.

\begin{tabular}{|c|c|c|c|c|c|c|c|c|}
\hline \multirow[b]{3}{*}{ DNA $\left(\frac{\text { copies }}{\text { reaction }}\right)$} & \multicolumn{4}{|c|}{ - Salmon DNA ${ }^{a}$} & \multicolumn{4}{|c|}{ + Salmon DNA ${ }^{\mathrm{b}}$} \\
\hline & \multicolumn{2}{|c|}{ WT template $(\mathrm{TTP} \pm \mathrm{SD})$} & \multicolumn{2}{|c|}{ MT template $(\mathrm{TTP} \pm \mathrm{SD})$} & \multicolumn{2}{|c|}{$\mathrm{WT}$ template $(\mathrm{TTP} \pm \mathrm{SD})$} & \multicolumn{2}{|c|}{ MT template $(\mathrm{TTP} \pm \mathrm{SD})$} \\
\hline & WT reaction & MT reaction & WT reaction & MT reaction & WT reaction & MT reaction & WT reaction & MT reaction \\
\hline $1 \times 10^{7}$ & $12.77 \pm 0.24$ & $17.62 \pm 0.32$ & $18.92 \pm 0.61$ & $13.29 \pm 0.25$ & $12.87 \pm 0.40$ & $19.19 \pm 0.63$ & $18.21 \pm 0.26$ & $13.33 \pm 0.60$ \\
\hline $1 \times 10^{6}$ & $14.67 \pm 0.22$ & $20.98 \pm 1.36$ & $22.21 \pm 2.18$ & $14.81 \pm 0.44$ & $14.75 \pm 0.28$ & $22.85 \pm 1.89$ & $21.27 \pm 0.61$ & $15.90 \pm 1.17$ \\
\hline $1 \times 10^{5}$ & $16.1 \pm 0.28$ & NEG & NEG & $16.81 \pm 0.27$ & $17.07 \pm 0.97$ & NEG & NEG & $17.23 \pm 1.07$ \\
\hline $1 \times 10^{4}$ & $18.57 \pm 0.64$ & NEG & NEG & $18.43 \pm 0.66$ & $20.22 \pm 1.30$ & NEG & NEG & $20.13 \pm 1.30$ \\
\hline $1 \times 10^{3}$ & $22.19 \pm 1.9$ & NEG & NEG & $21.54 \pm 1.79$ & $22.03 \pm 1.04$ & NEG & NEG & $23.96 \pm 1.38$ \\
\hline
\end{tabular}

Table 2. TTP values of standard curves of USS-sbLAMP reactions for detection of SNV p.H1047R. ${ }^{\text {aTTP values }}$ of WT and MT reactions with WT and MT template. ${ }^{\mathrm{b}} \mathrm{TTP}$ values of WT and MT reactions with WT and MT template, including Salmon DNA as background at $13 \mathrm{ng} /$ reaction. Reactions were considered as negative at TTP above $25 \mathrm{~min}$.

patient tumour tissue cores (Fig. 4d-f) were assessed as positive and negative controls for the p.H1047R mutation using the developed USS-sbLAMP assay. The T47D cell line DNA was analysed by Sanger sequencing (Fig. 4a), showing the high predominance of the nucleotide "G" over nucleotide "A". Amplification curves are shown in Fig. $4 \mathrm{~b}, \mathrm{c}$, corroborating the specificity and accuracy of the developed assay to amplify the corresponding target; the T47D cell line was uniquely amplified with the MT reaction indicating that the presence of the WT allele was out of the limit of detection to be amplified by the WT reaction. The MCF7 cell line was uniquely amplified with the WT reaction as expected. The obtained TTP values corresponded to approximately $1.90 \times 10^{3}$ copies/reaction (MCF7 cell line) and $1.53 \times 10^{4}$ copies/reaction (T47D cell line) according to the standard curves previously built for the WT and MT allele respectively. 


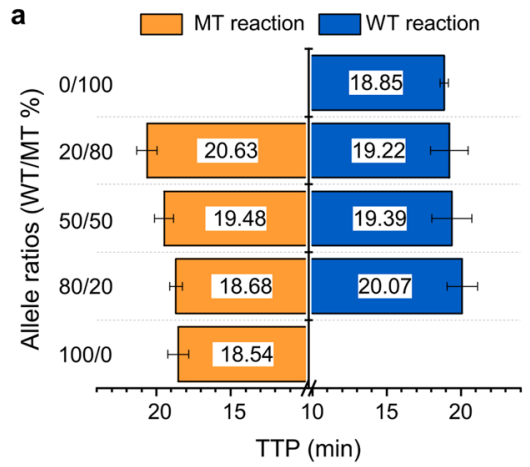

b

\begin{tabular}{|c|c|c|c|c|c|}
\hline \multicolumn{2}{|c|}{ Spiked DNA } & \multicolumn{2}{c|}{$\begin{array}{c}\text { Calculated concentration } \\
\text { (copies/reaction) }\end{array}$} & \multicolumn{2}{c|}{ Calculated ratios (\%) } \\
\hline WT (\%) & MT (\%) & WT allele & MT allele & WT allele & MT allele \\
\hline $\mathbf{0}$ & $\mathbf{1 0 0}$ & 0 & $1.67 \times 10^{4}$ & 0 & 100 \\
\hline $\mathbf{2 0}$ & $\mathbf{8 0}$ & $3.88 \times 10^{3}$ & $1.42 \times 10^{4}$ & 21 & 79 \\
\hline $\mathbf{5 0}$ & $\mathbf{5 0}$ & $7.72 \times 10^{3}$ & $5.69 \times 10^{3}$ & 58 & 42 \\
\hline $\mathbf{8 0}$ & $\mathbf{2 0}$ & $9.17 \times 10^{3}$ & $1.53 \times 10^{3}$ & 86 & 14 \\
\hline $\mathbf{1 0 0}$ & $\mathbf{0}$ & $1.33 \times 10^{4}$ & 0 & 100 & 0 \\
\hline
\end{tabular}

Figure 3. Study of SNV p.H1047R mix populations including wild-type and mutant alleles at different ratios. (a) TTP values of the spiked mixed populations at different ratios when tested with the WT and MT reactions, independently. (b) Calculated concentrations using the equations from the standard curves previously derived in Fig. 2, and calculated ratios using equation $\left(\frac{x_{1}}{x_{1}+y_{1}}\right) \times 100$ where $x_{1}$ and $y_{1}$ represent the calculated concentration of either the WT or MT allele. Mixtures were prepared to a final concentration of $10^{4}$ copies/ reaction $(100 \%)$. Spiked mixed populations at different ratios expressed in percentages $(100 / 0,80 / 20,50 / 50$, $20 / 80$, and $0 / 100)$.

DNA extracted from two patient FFPE tissue cores were used as controls for wild type (healthy tissue core) and heterozygous mutant (tumour tissue core) status respectively. Presence or absence of the p.H1047R mutation was confirmed by ddPCR (Fig. 4d). FFPE tissue DNA negative for the p.H1047R mutation (left hand panels in Fig. 4 d) showed an allele fraction of $0 \%$ c.3140 A > G MT allele. The heterozygous sample (right hand panels in Fig. 4d) showed an allele fraction of $38.2 \%$ c. $3140 \mathrm{~A}>\mathrm{G} \mathrm{MT}$ allele and $61.8 \%$ c.3140 A WT allele. The WT sample (FFPE tissue DNA negative for p.H1047R) uniquely amplified with the WT reaction (Fig. 4e), whilst the heterozygous sample (FFPE tissue DNA positive for p.H1047R) amplified with both reactions obtaining similar TTP values (Fig. 4f). The TTP values obtained with the FFPE tissue DNA WT sample corresponded to approximately $1.70 \times 10^{2}$ copies/reaction. In the case of the heterozygous sample, TTP values obtained with the WT reaction corresponded to $6.06 \times 10^{4}$ copies/reaction of the WT allele and TTP values obtained with the MT reaction corresponded to $1.04 \times 10^{5}$ copies/reaction of the MT allele. These results reinforce the capability of the developed assay to discriminate the SNV p.H1047R at isothermal conditions with high accuracy in FFPE tumour tissues, even in the presence of mixed populations.

Adaptation of SNV-specific LAMP on a Lab-on-Chip platform. The developed WT and MT reactions were tested using an ISFET-based Lab-on-Chip platform ${ }^{16,18}$ with synthetic DNA harbouring either the WT or MT allele to prove the feasible translation of the lab-based assay at a point-of-care setting. The reaction conditions were modified such that $\mathrm{pH}$ changes could occur during DNA amplification. This consisted of changing the buffering conditions in LAMP to those described in $\mathrm{pH}-\mathrm{LAMP}^{20}$. Each synthetic sample was tested with each of the WT and MT reactions independently for $25 \mathrm{~min}$ at a constant temperature of $63^{\circ} \mathrm{C}$ (provided by a thermal controller). As shown in Fig. 5, each reaction amplified the sample harbouring the targeted allele, as previously shown with the qPCR instrument. The amplification curves obtained on-chip are shown in Fig. 5b, and the TTP values were in agreement with the values obtained with the qPCR instrument. Besides the correlation of the TTP values, $\mathrm{pH}$ measurements of the reactions carried out on-chip and with the bulky instrument were also similar, as described in Fig. 5a. Consequently, we demonstrate a proof-of-concept that the developed assays can be safely transferred to the LoC platform for lab-free SNV detection of the mutation p.H1047R related to BC (Fig. 5).

\section{Discussion}

In this study, we report two novel primer sets using an allele-specific LAMP assay to detect the missense p.H1047R, PIK3CA somatic mutation, which is a common driver mutation in breast cancer. PIK3CA is also one of the most frequently mutated genes in human breast cancer and in numerous other malignancies ${ }^{24}$ due to the cellular importance of the PI3K signalling cascade. The potential clinical utility of being able to easily and rapidly determine PIK3CA mutations is yet to be fully explored. The SOLAR-1 trial recently presented that using combination therapy with Alpelisib for patients with an oncogenic PIK3CA mutation showed nearly double progression free survival ${ }^{25}$. Therefore, this assay could potentially be used to analyse diagnostic core biopsy tissue to identify a potential option for targeted monotherapy or combination therapies, with the recently approved small molecule inhibitor Alpelisib. Moreover, PIK3CA mutation is also associated with decreased effectivity of other common drugs such as Trastuzumab ${ }^{26}$. Therefore, detection of PIK3CA mutation could enable a precision medicine approach to determine the most appropriate therapy. These are two examples where a single mutation being identified can aid clinicians in their decision-making. Multiplexing this system where the LoC platform can detect panels of mutations would thus be a highly beneficial aid to clinicians in determining course of action as well as potentially monitoring MRD and relapse in BC patients.

The variant specific LAMP primer sets were designed to discriminate each allele by delaying or preventing unspecific amplification. At DNA concentrations below $1 \times 10^{6}$ copies/reaction of synthetic template, each 

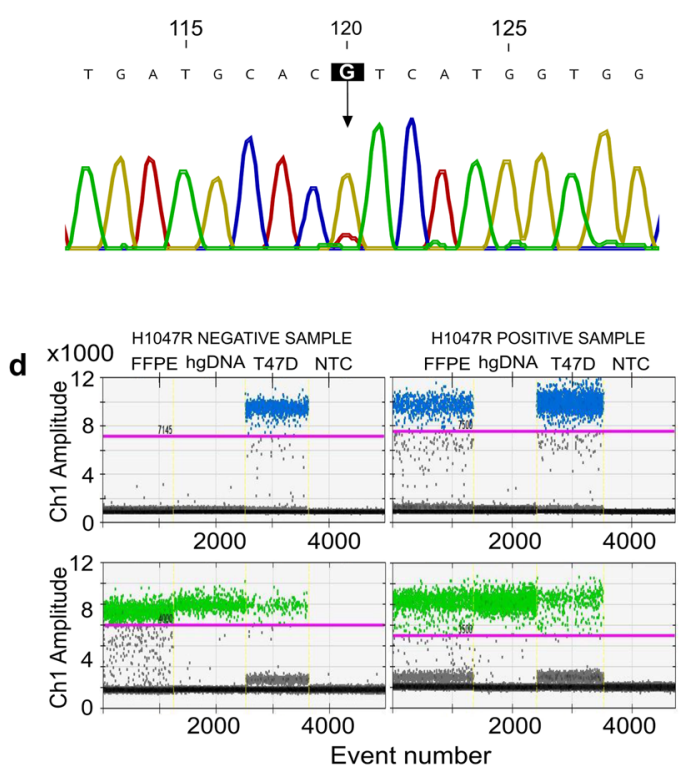

b
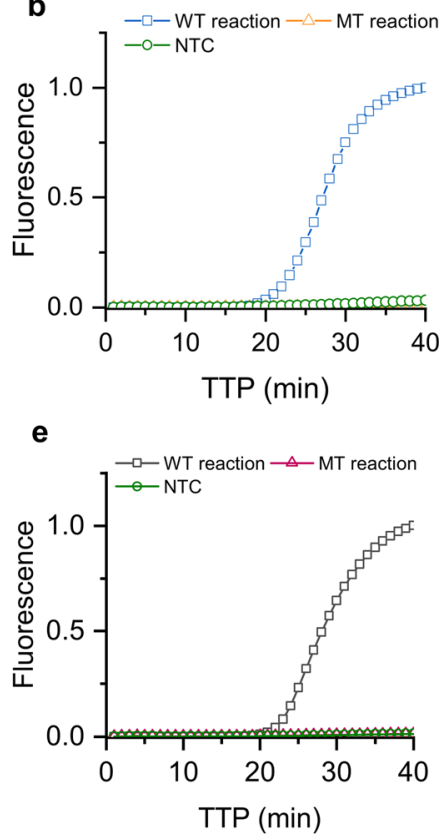

C

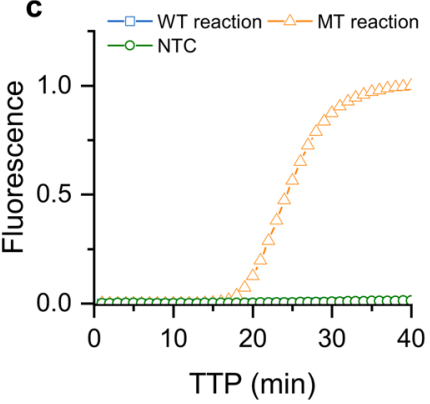

f

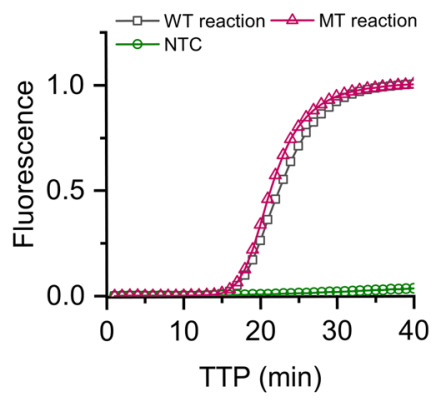

Figure 4. Amplification curves of USS-sbLAMP reactions for detection of SNV p.H1047R in T47D cell lines and Formalin-Fixed Paraffin-Embedded (FFPE) tissue. (a) Validation of p.H1047R mutation in T47D cell lines using Sanger Sequencing. High predominance of nucleotide "G" in the heterozygous cell line. (b) MCF7 cell line harbouring the WT p.H1047R allele tested with WT and MT reactions, independently. (c) T47D heterozygous cell line tested with WT and MT reactions, independently. (d) ddPCR validation of p.H1047R mutation in FFPE tissue derived DNA samples. DNA samples included $10 \mathrm{ng}$ of FFPE DNA (FFPE), $10 \mathrm{ng}$ of hgDNA as negative control (hgDNA), $10 \mathrm{ng}$ of T47D cell line as heterozygous control (T47D) and no template control (NTC). Representative one-dimensional (1D) amplitude plots are shown with the associated manual threshold gating (pink line) for FAM (mutant, blue) amplitudes in upper panels and VIC (wild type, green) amplitudes in lower panels. Black dots correspond to droplets without any dye fluorescence. Left hand panel shows FFPE tissue DNA negative for c.3140 A > G mutation and right-hand panel shows FFPE tissue DNA heterozygous for the c.3140 A > G mutation. (e) FFPE tissue DNA harbouring the WT p.H1047R allele was tested with WT and MT reactions, independently. (f) FFPE tissue heterozygous DNA harbouring both WT and MT alleles was tested with WT and MT reactions, independently.

a

\begin{tabular}{|c|c|c|c|c|c|}
\hline \multirow{2}{*}{ Allele } & \multirow{2}{*}{ Reaction } & \multicolumn{2}{|c|}{ TTP (min) } & \multicolumn{2}{c|}{$\Delta p H$ (pH units) } \\
\cline { 3 - 6 } & & LoC & qPCR & LoC $^{\text {a }}$ & qPCR $^{\text {b }}$ \\
\hline WT & WT & 18.5 & 18.3 & 0.9 & 0.9 \\
\hline WT & MT & $>25$ & NEG & 0.1 & 0.2 \\
\hline MT & WT & $>25$ & NEG & 0.1 & 0.1 \\
\hline MT & MT & 23.2 & 19.0 & 0.5 & 1.0 \\
\hline
\end{tabular}

b

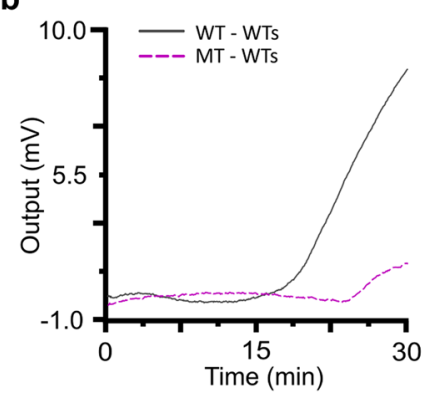

c

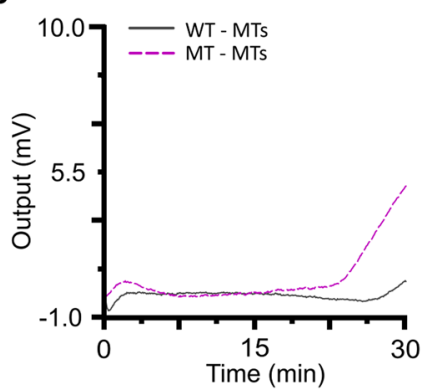

Figure 5. Lab-on-Chip results for the detection of SNV p.H1047R. (a) TTP values and $\Delta \mathrm{pH}$ measurements of reactions performed on the Lab-on-Chip platform and conventional qPCR instrument. (b) Amplification curves of the WT template with WT and MT reactions carried-out independently on the Lab-on-Chip platform. (c) Amplification curves of the MT template with WT and MT reactions carried-out independently on the Labon-Chip platform. ${ }^{\mathrm{a}}$ Equivalent $\mathrm{pH}$ based on microchip sensitivity $(-9.23 \mathrm{mV} / \mathrm{pH}) .{ }^{\mathrm{b}} \mathrm{pH}$ measurements with a pH-meter (Sentron).

primer set uniquely amplified the targeted allele. Analytical specificity and sensitivity were preserved in the presence of background Salmon DNA with a limit of detection of $1 \times 10^{3}$ copies/reaction, which equates to $0.003 \mathrm{pg} /$ reaction for the synthetic template. Furthermore, allele discrimination within allelic ratios in spiked mixed populations was demonstrated enabling quantification with values within $8 \%$ from the reference ones. As a proof-of-concept, DNA samples from two breast cancer cell lines and FFPE tumour tissues were tested with the developed assay. The results obtained, reinforced the robustness, sensitivity and specificity of the developed 
assay for the discrimination of p.H1047R in cell lines and patient tissue samples. In addition, we show allelic discrimination using a $\mathrm{pH}$ sensitive variation of the proposed assay with a CMOS microchip (Fig. 5) ${ }^{16}$. The microchip consisted of an array of ISFET sensors which detected both alleles with a positive change in $\mathrm{pH}$ of around $1-1.48 \mathrm{pH}$ units. Overall, we show novel sbLAMP primer sets with the potential to discriminate BC tissue samples carrying the mutation p.H1047R as well as detection with CMOS-based ISFET sensors. This may provide the basis to develop an alternative to blood-based mutation testing by NGS and ddPCR technology in a quicker and more affordable way for use with plasma-derived cfDNA.

Other studies have reported the capability of detecting the mutation p.H1047R. Alvarez-Garcia et al. ${ }^{27}$ genotyped PIK3CA status with an analytical sensitivity of $56 \mathrm{pg}$ (9 genome equivalents) using qPCR technology and chemically modified primers. In a similar way, Wang et al. ${ }^{28}$ used non-extendable primer blockers for allele-specific PCR detection of three mutations in cancer $K-R A S, B-R A F$, and EGFR with a limit of detection of a single copy. A different approach reported by Gyanchandani et al. ${ }^{29}$, has been used to demonstrate amplification of cfDNA from liquid biopsies linked to metastatic BC without compromising allelic balance. This step enriched the cfDNA sample, allowing ddPCR and sequencing to become more feasible due to the higher amount of DNA template required. Numerous NGS technologies such as BEAMing and Safe-SeqS have been used to detect known breast cancer mutations using ctDNA with sensitivity higher than $99 \%{ }^{30}$. Our own studies have shown for primary patients that recurred, patient specific ctDNA profiling detected molecular relapse up to 2 years ahead of clinical relapse with $89 \%$ sensitivity and $100 \%$ specificity ${ }^{31}$. However, despite its high sensitivity and specificity, NGS platforms are still costly and require specialist time for data analysis and experimentation. As such, NGS can be useful to discover tumour mutations that can aid in treatment decisions and cancer classifications but this technique is not currently used routinely to monitor cancer response to treatment. On the other hand, our isothermal assay and Lab-on-Chip platform may allow for rapid, affordable and portable monitoring of individual breast cancer mutations without the need of thermal cycling, chemically modified primers or bulky and expensive equipment. Going forward, the next step will be to develop a multiplex assay to survey several common breast cancer mutations. The cost-effectiveness of such a test will allow breast cancer to be monitored routinely during patient's therapeutic journey.

LAMP has been previously used to detect mutations in tumour tissue such as for KRAS mutations in colorectal colon cancer ${ }^{32}$ using LAMP in tandem with ligation substrates. Two studies specific to the detection of PIK3CA mutations published recently involved a colorimetric assay using strand displacement amplification ${ }^{33}$, and recombinase polymerase amplification (RPA), which is another isothermal amplification method ${ }^{34}$. These other studies highlight the demand for a cost-effective and rapid method for mutational tracking of DNA markers in cancer. In this paper, we demonstrate the genotyping of breast cancer variants with isothermal methods and CMOS technology, specifically detecting the PIK3CA p.H1047R variant. The combination of these principles indicates the feasibility of a label free Lab-on-Chip platform that can genotype breast cancer variants rapidly to assist tumour progression surveillance and individuality of BC in the clinic as an affordable alternative to NGS and ddPCR.

\section{Materials and Methods}

Samples and DNA extraction methods. Two plasmids, purchased from ThermoFisher Scientific (United States), were utilised as the synthetic DNA material for validation of the developed primer sets. Both plasmids consisted of PUC 18 cloning vectors containing a fragment of interest of 531 bp harbouring the SNV p.H1047R. The plasmid harbouring the WT allele p.H1047R is named as WT template, and the plasmid harbouring the MT allele p.H1047R is named as MT template. On the other hand, DNA was extracted from patient FFPE tissue cores using the Qiagen GeneRead Kit according to manufacturer's instructions and quantified using Qubit fluorometer. DNA was extracted from a healthy control tissue core as a WT control (H1047R negative) and from a tumour patient core (H1047R heterozygous sample). Patient samples used in this study were from our recent paper Coombes et al. ${ }^{31}$ with the trial protocol approved by the Riverside Research Ethics Committee REC:13/ LO/115; IRAS:126462. Methods were carried out in accordance with the relevant guidelines and regulations. Informed consent was obtained from all participants and/or their legal guardian/s.

Sanger sequencing. The T47D cell line was genotyped by PCR amplification using 30 ng of DNA, followed by Sanger Sequencing using an ABI 3730 automatic genetic analyser (Applied Biosystems, Foster City, CA). Primers sequences included: forward, 5' - AGAACTACAATCTTTTGATGACA - $3^{\prime}$, and reverse, $5^{\prime}$-TGGAATCCAGAGTGAGCT-3'. The sequence reads were analysed using the sequencing analysis tools in Geneious ${ }^{35}$.

Droplet Digital PCR. Validation of the PIK3CA p.H1047R mutation was performed using a Bio-Rad QX200 droplet digital PCR system as described previously ${ }^{36}$. Primer sequences included: forward, 5'-AGAGGCTTTGGAGTATTTCATG-3'; reverse, 5' -TGCATGCTGTTTAATTGTGTG-3'; probe sequences were wild-type VIC-MGB 5'-CCACCATGATGTGCA- $3^{\prime}$; and mutant FAM-MGB 5'-CCACCATGACGTGCA- $3^{\prime}$. The optimum melting temperature for this assay was $62^{\circ} \mathrm{C}$. The assay was designed using OligoArchitect. For each assay $10 \mathrm{ng}$ of FFPE tissue DNA was run alongside $10 \mathrm{ng}$ of hgDNA (negative control), $10 \mathrm{ng}$ TD47 cell line DNA (heterozygous control) and NTC (no template control).

SNV-specific primer design for detecting p.H1047R. Consensus reference genomic sequence from human gene PIK3CA (Gene ID: 5290) was retrieved from National Centre for Biotechnology Information $(\mathrm{NCBI})^{37}$ and analysed for specific target regions among biological sequences using BLAST ${ }^{38}$. Following the guidelines provided by Malpartida-Cardenas et al. ${ }^{15}$ for the design of USS-sbLAMP primers, two primer sets were designed targeting the SNV p.H1047R within the gene PIK3CA. Each primer set consisted of 8 primers, being 6 of them responsible for SNV-based loop mediated isothermal amplification (sbLAMP) including sbFIP and sbBIP which are in charge of the allele-specificity, and 2 unmodified self-stabilising (USS) primers, FB and 
$\mathrm{BB}$, responsible for delaying or preventing unspecific sbLAMP amplification. The sbLAMP primers were designed using Primer Explorer V5 (Eiken Chemical Co. Ltd., Tokyo, Japan; http://primerexplorer.jp/lampv5e/index.html) and LAMP guidelines ${ }^{14}$ to obtain F3, B3, F2 and B2 priming regions, and optimized manually to locate both loop primers LF and LB, and the SNV at the $5^{\prime}$ end of F1c and B1c within sbFIP and sbBIP. USS primers were designed following the method described by Malpartida-Cardenas et al. ${ }^{15}$ based on the most optimal sbFIP and sbBIP tested experimentally. The primer set specifically detecting the WT allele p.1047 H, named as USS $\mathrm{MT}^{- \text {sbLAMP }_{\mathrm{WT}}}$

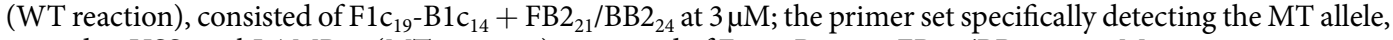
named as $\mathrm{USS}_{\mathrm{WT}}-\mathrm{SbLAMP}_{\mathrm{MT}}$ (MT reaction), consisted of $\mathrm{F}_{1 \mathrm{c}}-\mathrm{B} 1 \mathrm{c}_{14}+\mathrm{FB} 2_{19} / \mathrm{BB}_{23}$ at $3 \mu \mathrm{M}$.

Reaction conditions. Each LAMP reaction mixture contained the following: $1.5 \mu \mathrm{L}$ of 10x isothermal buffer, $0.9 \mu \mathrm{L}$ of MgSO 4 ( $100 \mathrm{mM}$ stock), $2.1 \mu \mathrm{L}$ of dNTPs ( $10 \mathrm{mM}$ stock), $0.375 \mu \mathrm{L}$ of BSA $(20 \mathrm{mg} / \mathrm{mL}$ stock), $2.4 \mu \mathrm{L}$ of Betaine ( $5 \mathrm{M}$ stock), $0.375 \mu \mathrm{L}$ of SYTO 9 Green $(20 \mu \mathrm{M}$ stock), $0.6 \mu \mathrm{L}$ of Bst 2.0 DNA polymerase $(8,000 \mathrm{U} / \mathrm{mL}$ stock), $3 \mu \mathrm{L}$ of different concentrations of synthetic plasmid DNA, $1.5 \mu \mathrm{L}$ of $10 x$ USS-sbLAMP primer mixture $(20 \mu \mathrm{M}$ sbBIP/sbFIP, $10 \mu \mathrm{M} \mathrm{LF} / \mathrm{LB}, 2.5 \mu \mathrm{M} \mathrm{B} 3 / \mathrm{F} 3$ and $30 \mu \mathrm{M}$ of FB/BB) and enough nuclease-free water (ThermoFisher Scientific) to bring the volume to $15 \mu \mathrm{L}$. All reagents were purchased from New England BioLabs (United Kingdom). Reactions were performed at $63^{\circ} \mathrm{C}$ for $35 \mathrm{~min}$. Two reactions, WT reaction and MT reaction, were performed independently. Experiments were performed twice with each condition in triplicate $(5 \mu \mathrm{L}$ each reaction) utilising LightCycler 480 Multiwell Plates 96 (Roche Diagnostics) and a LightCycler 96 Real-Time PCR System (Roche Diagnostics. In the case of $\mathrm{pH}$-LAMP, the buffering conditions were modified such that $\mathrm{pH}$ changes could be measured. Each reaction contained the following: $3.0 \mu \mathrm{L}$ of $10 \mathrm{x}$ isothermal customized buffer (pH 8.5-9), $1.8 \mu \mathrm{L}$ of MgSO 4 (100 mM stock), $4.2 \mu \mathrm{L}$ of dNTPs (10 mM stock), $1.8 \mu \mathrm{L}$ of BSA ( $20 \mathrm{mg} / \mathrm{mL}$ stock), $4.8 \mu \mathrm{L}$ of Betaine ( $5 \mathrm{M}$ stock), $1.88 \mu \mathrm{L}$ of Bst $2.0 \mathrm{DNA}$ polymerase $(8,000 \mathrm{U} / \mathrm{mL}$ stock), $3 \mu \mathrm{L}$ of different concentrations of synthetic DNA or gDNA, $0.75 \mu \mathrm{L}$ of $\mathrm{NaOH}(0.2 \mathrm{M}$ stock), $3 \mu \mathrm{L}$ of $10 \mathrm{x}$ LAMP primer mixture, $0.75 \mu \mathrm{L}$ of SYTO 9 Green $(20 \mu \mathrm{M}$ stock) only for qPCR experiments, and enough nuclease-free water (ThermoFisher Scientific) to bring the volume to $30 \mu \mathrm{L}$. Reactions tested on the microchip contained $13 \mu \mathrm{L}$ each. sbLAMP and USS primers were purchased from Integrated DNA Technologies (The Netherlands) and resuspended in TE buffer to $100 \mu \mathrm{M}$ and $400 \mu \mathrm{M}$ stock solutions, respectively. The solutions were stored at $4^{\circ} \mathrm{C}$.

Analytical sensitivity and specificity of USS-sbLAMP primer sets for detection of SNV p.H1047R. Analytical sensitivity was tested using ten-fold serial dilutions $\left(1 \times 10^{7}, 1 \times 10^{6}, 1 \times 10^{5}, 1 \times 10^{4}\right.$ and $1 \times 10^{3}$ copies/reaction which are equivalent to 31.356, 3.136, 0.314, 0.031 and $0.003 \mathrm{pg} / \mathrm{reaction}$ ) of WT and MT templates, independently. Standard curves were obtained by plotting the TTP values with errors at one standard deviation against their corresponding DNA concentrations. Sensitivity including background DNA was also assessed by including in the reaction mixture Salmon testes sonicated DNA (Sigma-Aldrich) at $13 \mathrm{ng} / \mathrm{reaction}$. Sensitivity of mixed populations was evaluated by spiking WT and MT templates at different ratios $(100 / 0,80 / 20$, $50 / 50,20 / 80$ and $0 / 100$, in percentage) to a final concentration of $1 \times 10^{7}$ copies/reaction.

SNV-specific LAMP on a microchip-based Lab-on-Chip platform. Electrochemical sensing for the detection of the $\mathrm{pH}$ changes induced during $\mathrm{pH}$-LAMP is facilitated using ISFETs fabricated in unmodified complementary metal-oxide-semiconductor (CMOS) technology. ISFETs detect changes in the concentration of hydrogen ions at the passivation layer of the microchip through the modulation of an induced voltage across that layer ${ }^{22,39}$. This way, owing to the compatibility of fabrication in standard CMOS technology allows for the mass manufacturing, low cost, and miniaturisation of sensors while ensuring non-optical and fully-electronic chemical ( $\mathrm{pH}$ ) detection.

The Lab-on-Chip platform used here comprises an array of $64 \times 64$ ISFET sensors fabricated in the AMS 0.35 $\boldsymbol{\mu} \boldsymbol{m}$ CMOS process using silicon nitride $\left(\boldsymbol{S i}_{3} \boldsymbol{N}_{4}\right)$ as the passivation (sensing) layer. The total sensing area spans 0.56 $\mathbf{m m}^{2}$ with an input-output $\mathrm{pH}$ sensitivity of $9.23 \mathrm{mV} / \mathrm{pH}$. A detailed description of the circuit characteristics and additional specification is provided in N. Miscourides et al. ${ }^{16}$. Furthermore, a custom microfluidic manifold was added on top of the microchip to host the reaction mix during LAMP, laser cut from plastic acrylic sheets. Thermal control for DNA amplification is facilitated using an external Verity Thermal Cycler instrument.

Statistical analysis. Data is presented in minutes as TTP \pm error at one standard deviation. Displayed p-values were calculated using a two-sided distribution Student's heteroscedastic t-test; statistical significance was considered as following: ${ }^{*}$ p-value $<0.05, * *$ p-value $<0.01$, ***p-value $<0.001$, ****p-value $<0.0001$.

\section{Data availability}

All data generated or analysed during this study are included in this published article. Data generated and analysed throughout the study can be made available from the corresponding author on reasonable request.

Received: 26 September 2019; Accepted: 13 February 2020;

Published online: 12 March 2020

\section{References}

1. Early Breast Cancer Trialists' Collaborative Group (EBCTCG). Effects of chemotherapy and hormonal therapy for early breast cancer on recurrence and 15-year survival: an overview of the randomised trials. Lancet. 365, 1687-1717 (2005).

2. UK, C. R. Breast cancer incidence (invasive) statistics. (2015).

3. Beca, F. \& Polyak, K. Intratumor Heterogeneity in Breast Cancer. Adv. Exp. Med. Biol. 882, 169-189 (2016).

4. Kabel, A. M. Tumor markers of breast cancer: New prospectives. J. Oncol. Sci. 3, 5-11 (2017).

5. Shaw, J. A. et al. Mutation Analysis of Cell-Free DNA and Single Circulating Tumor Cells in Metastatic Breast Cancer Patients with High Circulating Tumor Cell Counts. Clin. Cancer Res. 23, 88-96 (2017). 
6. Saal, L. H. et al. PIK3CA mutations correlate with hormone receptors, node metastasis, and ERBB2, and are mutually exclusive with PTEN loss in human breast carcinoma. Cancer Res 65, 2554-2559 (2005).

7. Costa, R. L. B., Han, H. S. \& Gradishar, W. J. Targeting the PI3K/AKT/mTOR pathway in triple-negative breast cancer: a review. Breast Cancer Res Treat 169, 397-406 (2018).

8. Mangone, F. R., Bobrovnitchaia, I. G., Salaorni, S., Manuli, E. \& Nagai, M. A. PIK3CA exon 20 mutations are associated with poor prognosis in breast cancer patients. Clin. (Sao Paulo) 67, 1285-1290 (2012).

9. Richardson, A. L. \& Iglehart, J. D. BEAMing Up Personalized Medicine: Mutation Detection in Blood. Clin. Cancer Res. 18, 3209-3211 (2012).

10. Higgins, M. J. et al. Detection of Tumor PIK3CA Status in Metastatic Breast Cancer Using Peripheral Blood. Clin. Cancer Res. 18, 3462-3469 (2012).

11. Guttery, D. S. et al. Hide and seek: tell-tale signs of breast cancer lurking in the blood. Cancer Metastasis Rev. 32, 289-302 (2013).

12. Garcia-Murillas, I. et al. Mutation tracking in circulating tumor DNA predicts relapse in early breast cancer. Sci. Transl. Med. 7, 302ra133-302ra133 (2015)

13. Page, K. et al. Next Generation Sequencing of Circulating Cell-Free DNA for Evaluating Mutations and Gene Amplification in Metastatic Breast Cancer. Clin. Chem. 63, 532-541 (2017).

14. Notomi, T. et al. Loop-mediated isothermal amplification of DNA. Nucleic Acids Res. 28, E63 (2000).

15. Malpartida-Cardenas, K. et al. Allele-Specific Isothermal Amplification Method Using Unmodified Self-Stabilizing Competitive Primers. Anal. Chem. 90, 11972-11980 (2018).

16. Miscourides, N. \& Georgiou, P. ISFET Arrays in CMOS: A Head-to-Head Comparison between Voltage and Current Mode. IEEE Sens. J. 19, 1224-1238 (2019).

17. Malpartida-Cardenas, K. et al. Quantitative and rapid Plasmodium falciparum malaria diagnosis and artemisinin-resistance detection using a CMOS Lab-on-Chip platform. bioRxiv (2019).

18. Miscourides, N., Yu, L.-S., Rodriguez-Manzano, J. \& Georgiou, P. A 12.8 k Current-Mode Velocity-Saturation ISFET Array for OnChip Real-Time DNA Detection. IEEE Trans. Biomed. Circuits Syst. 1-13, https://doi.org/10.1109/TBCAS.2018.2851448 (2018).

19. Moser, N., Rodriguez-Manzano, J., Lande, T. S. \& Georgiou, P. A Scalable ISFET Sensing and Memory Array With Sensor AutoCalibration for On-Chip Real-Time DNA Detection. IEEE Trans. Biomed. Circuits Syst. 1-12, https://doi.org/10.1109/ TBCAS.2017.2789161 (2018).

20. Toumazou, C. et al. Simultaneous DNA amplification and detection using a pH-sensing semiconductor system. Nat. Methods 10, 641-6 (2013).

21. Kalofonou, M. \& Toumazou, C. An ISFET based analogue ratiometric method for DNA methylation detection. in 2014 IEEE International Symposium on Circuits and Systems (ISCAS) 1832-1835 https://doi.org/10.1109/ISCAS.2014.6865514 (IEEE, 2014).

22. Kalofonou, M. \& Toumazou, C. Semiconductor technology for early detection of DNA methylation for cancer: From concept to practice. Sensors Actuators B Chem. 178, 572-580 (2013).

23. Kalofonou, M. \& Toumazou, C. A low power sub- $\mu \mathrm{W}$ chemical gilbert cell for ISFET differential reaction monitoring. IEEE Trans. Biomed. Circuits Syst. 8, 565-574 (2014).

24. Samuels, Y. \& Waldman, T. In 21-41 https://doi.org/10.1007/82_2010_68 (2010).

25. André, F. et al. Alpelisib for PIK3CA -Mutated, Hormone Receptor-Positive Advanced Breast Cancer. N. Engl. J. Med. 380, 1929-1940 (2019).

26. Berns, K. et al. A Functional Genetic Approach Identifies the PI3K Pathway as a Major Determinant of Trastuzumab Resistance in Breast Cancer. Cancer Cell 12, 395-402 (2007).

27. Alvarez-Garcia, V. et al. A simple and robust real-time qPCR method for the detection of PIK3CA mutations. Sci. Rep. 8, 4290 (2018).

28. Wang, H. et al. Allele-Specific, Non-Extendable Primer Blocker PCR (AS-NEPB-PCR) for DNA Mutation Detection in Cancer. J. Mol. Diagnostics 15, 62-69 (2013).

29. Gyanchandani, R. et al. Whole genome amplification of cell-free DNA enables detection of circulating tumor DNA mutations from fingerstick capillary blood. Sci. Rep. 8, 17313 (2018).

30. Elazezy, M. \& Joosse, S. A. Techniques of using circulating tumor DNA as a liquid biopsy component in cancer management. Comput. Struct. Biotechnol. J. 16, 370-378 (2018).

31. Coombes, R. C. et al. Personalized Detection of Circulating Tumor DNA Antedates Breast Cancer Metastatic Recurrence. Clin. Cancer Res. 25, 4255-4263 (2019).

32. Fu, Y. et al. Detection of KRAS mutation via ligation-initiated LAMP reaction. Sci. Rep. 9, 5955 (2019).

33. Shen, C. et al. High-sensitive colorimetric biosensing of PIK3CA gene mutation based on mismatched ligation-triggered cascade strand displacement amplification. Sensors Actuators B Chem. 273, 377-383 (2018).

34. Martorell, S., Palanca, S., Maquieira, Á. \& Tortajada-Genaro, L. A. Blocked recombinase polymerase amplification for mutation analysis of PIK3CA gene. Anal. Biochem. 544, 49-56 (2018).

35. Kearse, M. et al. Geneious Basic: An integrated and extendable desktop software platform for the organization and analysis of sequence data. Bioinformatics 28, 1647-1649 (2012).

36. Myint, N. N. M. et al. Circulating tumor DNA in patients with colorectal adenomas: assessment of detectability and genetic heterogeneity. Cell Death Dis. 9, 894 (2018).

37. National Center for Biotechnolgy Information (NCBI). National Center for Biotechnolgy Information (NCBI). Available at, https:// www.ncbi.nlm.nih.gov/.

38. Altschul, S. F., Gish, W., Miller, W., Myers, E. W. \& Lipman, D. J. Basic local alignment search tool. J. Mol. Biol. 215, $403-410$ (1990).

39. Moser, N., Lande, T. S., Toumazou, C. \& Georgiou, P. ISFETs in CMOS and Emergent Trends in Instrumentation: A Review. IEEE Sens. J. 16, 6496-6514 (2016).

\section{Acknowledgements}

This work was supported by the Cancer Research UK - Multidisciplinary Award [C54044/A25292], Science Committee Programme Award [C14315/A23464] and the EPSRC HiPEDS CDT [EP/L016796/1]. We thank the Imperial Cancer Research UK Centre, Experimental Cancer Medicine Centre (ECMC) and the Imperial College Tissue Bank for supporting patient recruitment and sample collection.

\section{Author contributions}

M.K., J.R.-M. and C.T. conceived the idea. Experiments were designed and analysed by J.R.-M., K.M.-C. and M.K. K.M.-C., G.A., L.-S.Y., N.M., J.R.-M and M.K. were involved in conducting the experiments and the analysis of the data. R.A. and D.F.G. were involved in conducting some experiments and analysis of ddPCR data. J.S., R.C.C. and S.A. provided the DNA samples and reviewed the standardisation protocols. K.L.T.G., K.G., K.P. supported patient recruitment, sample collection and DNA isolation. N.M. and P.G. provided the ISFET arrays for the conducted experiments. K.M.-C., N.M., G.A., J.R.-M., and M.K. were involved in writing the paper and all authors reviewed and approved the final manuscript. 


\section{Competing interests}

The authors declare no competing interests.

\section{Additional information}

Correspondence and requests for materials should be addressed to M.K.

Reprints and permissions information is available at www.nature.com/reprints.

Publisher's note Springer Nature remains neutral with regard to jurisdictional claims in published maps and institutional affiliations.

(c) (i) Open Access This article is licensed under a Creative Commons Attribution 4.0 International License, which permits use, sharing, adaptation, distribution and reproduction in any medium or format, as long as you give appropriate credit to the original author(s) and the source, provide a link to the Creative Commons licence, and indicate if changes were made. The images or other third party material in this article are included in the article's Creative Commons licence, unless indicated otherwise in a credit line to the material. If material is not included in the article's Creative Commons licence and your intended use is not permitted by statutory regulation or exceeds the permitted use, you will need to obtain permission directly from the copyright holder. To view a copy of this licence, visit http://creativecommons.org/licenses/by/4.0/.

(c) The Author(s) 2020 